\title{
Estructura vertical e índice de valor forestal ecológico de la vegetación arbórea del Bosque Reservado en Tingo María, 2021
}

Vertical structure and ecological forest value index of the arboreal vegetation of the Reserve Forest in Tingo María, 2021

\author{
Luis Eduardo Oré Cierto \\ Universidad Nacional Agraria de la Selva \\ luisore21793@gmail.com \\ Edilberto Díaz Quintana \\ Universidad Nacional Agraria de la Selva \\ edilberto.diaz@unas.edu.pe \\ Wendy Caroline Loarte Aliaga \\ Universidad de Huánuco \\ wendy_loarte_aliaga@outlook.com.pe
}

\begin{abstract}
RESUMEN
La investigación tuvo por objetivo determinar la estructura vertical y el índice del valor forestal (IVF) en el bosque de la Universidad Nacional Agraria de la Selva - Departamento Huánuco; se trabajó con dos parcelas permanentes de medición (PPM-1, PPM-4) de 1,0 ha., y se midieron: diámetro a la altura del pecho (dap), altura total y diámetro de la copa de las especies con dap $\geq 10 \mathrm{~cm}$.; como resultado la PPM-1 presentó 88 individuos en el estrato bajo, arboles menores a 11,45 m de altura; 457 y 101 individuos en el estrato medio y alto, arboles mayores a 22,9 m de altura, siendo la especie con mayor índice de espacio vital (IEV) la Miconia punctata $(0,98)$ y la especie con mayor posición sociológica (PS) la Senefeldera inclinata $(10,02 \%)$, y la estructura bidimensional EB), la Parkia panurensis registró el 39,73\% del IVF; la PPM-4 presentó 98 individuos en el estrato bajo, arboles menores a 13,00 m de altura; 371 y 75 individuos en el estrato medio y alto, arboles mayores a 26,00 m de altura, siendo la especie con mayor IEV la Hirtella racemosa $(0,79)$, la especie con mayor PS fue la Senefeldera inclinata (31,90\%), y la EB registró el 68,28\% del IVF.
\end{abstract}

Palabras clave: Estructura vertical, índice de valor forestal, Índice de espacio vital, bidimensional. 


\begin{abstract}
The objective of the research was to determine the vertical structure and the forest value index (IVF) in the forest of the National Agrarian University of La Selva - Huánuco Department; We worked with two permanent measurement plots (PPM-1, PPM-4) of 1.0 ha., and were measured: diameter at breast height (dbh), total height and crown diameter of the species with $\mathrm{dbh} \geq 10 \mathrm{~cm}$; As a result, the PPM-1 presented 88 individuals in the low stratum, trees less than 11,45 m in height; 457 and 101 individuals in the middle and upper stratum, trees greater than 22,9 $\mathrm{m}$ in height, the species with the highest index of living space (IEV) being Miconia punctata $(0,98)$ and the species with the highest sociological position (PS ) Senefeldera inclinata (10,02\%), and the twodimensional structure EB), Parkia panurensis registered 39,73\% of the IVF; PPM-4 presented 98 individuals in the low stratum, trees less than 13,00 m in height; 371 and 75 individuals in the middle and upper stratum, trees greater than $26,00 \mathrm{~m}$ in height, the species with the highest IEV being Hirtella racemosa $(0,79)$, the species with the highest SP was Senefeldera inclinata (31,90\%), and the EB registered 68,28\% of the IVF.
\end{abstract}

Keywords: Vertical structure, forest value index, Living space index, two-dimensional

\section{INTRODUCCIÓN}

El alto grado de deterioro que presentan las masas forestales de los bosques amazónicos de Perú es por causa de la agricultura migratoria, ejercido por agricultores y/o campesinos que queman al año entre 1 y 2 hectáreas aproximadamente para plantar sus cultivos, a ello le sigue la agricultura extensiva de cultivos como la palma aceitera, los cultivos ilictos de hoja de coca, la tala ilegal y la minería ilegal (Gestión, 2020). También en el informe del Proyecto Monitoreo de la Amazonía Andina (MAAP) revela que del 2013 al 2015 se deforestaron cerca de 25 mil hectáreas de bosque en la zona este de la región Huánuco. La causa sería la habilitación de áreas para el cultivo de pasto para el ganado (Servindi, 2016). Además de acuerdo con O’Hara et al. (1995), indica que la distribución vertical del dosel de los árboles es una característica de la estructura forestal siendo importante para el manejo de los recursos forestales, los recursos hídricos, los aspectos relacionados con la estética, con el crecimiento y con la producción de los bosques; y Valerio y Salas (1997) indica que la distribución vertical responde a las características de las especies que la conforman y a las condiciones micro climáticas que varían al moverse de arriba abajo en el perfil: radiación, temperatura, viento, humedad relativa, evapotranspiración y concentración de CO2, Ramos (2004) añade que, está relacionada a la distribución de biomasa en el plano vertical, es decir, a la distribución de organismos a lo alto de su perfil, permitiendo esta diferencia de microambientes que las especies se ubiquen en los diferentes niveles en función de satisfacer de mejor 
manera sus requerimientos de energía, siendo una variable básica a tomar en cuenta, a nivel local, la posición social de la copa, refiriéndose esta al acceso a luz que tenga un individuo, Krebs (1986) indica que, la estructura vertical está asociada con una disminución de la luminosidad, donde la competencia por la luz es un factor crítico en la determinación de la estratificación del bosque. La luz es un recurso muy importante y la competencia entre plantas para llegar a la luz es fuerte, la planta ganadora no es necesariamente la planta con mayor follaje, sino aquella cuyo follaje presenta la mejor disposición para interceptar la luz, la altura de la planta juega un papel muy importante para la captación de luz. Corella et al. (2001) indica que el índice de valor forestal y/o estructura bidimensional refieren tres medidas: la primera al nivel del estrato inferior en el plano horizontal (diámetro a la altura del pecho), la segunda que incluye los estratos inferior y superior en el plano vertical (altura), la tercera al nivel del estrato superior en el plano horizontal (cobertura).

La determinación de la condición actual del bosque se ha extendido a varias disciplinas de las ciencias naturales, pero el estudio de su distribución y estructura son los de mayor impacto (Baró et al., 2014; Burkhart \& Tomé, 2012; Savard et al., 2000). Los beneficios que aportan la vegetación, está directamente relacionados con su composición actual (Saavedra-Romero et al., 2016), sus dimensiones (Li et al., 2011; Troxel et al., 2013), y cobertura (Mori et al., 2015; Schomaker et al., 2007), por lo que el valor ecosistémico aumenta debido a la eficiencia en la captura de contaminantes; por lo que su capacidad de interceptar y absorber contaminantes depende de la especie forestal, volumen de sus copas (Korhonen et al., 2013), área superficial e índice de área foliar, topografía foliar (Janhäll, 2015) y, el grupo arbóreo (Beckett et al., 2000; Räsänen et al., 2013); Nowak \& Heisler (2010) menciona que los árboles sanos de más de $75 \mathrm{~cm}$ de diámetro eliminan 60 a 70 veces más contaminantes atmosféricos al año $(1.4 \mathrm{~kg})$ que los árboles pequeños menores a $7 \mathrm{~cm}$ de diámetro $(0.025$ $\mathrm{kg})$.

Posterior a la intervención de los bosques, se observa la gran capacidad de resiliencia de los mismos (Guerra et al., 2020), en donde en las áreas aperturadas proliferan especies de rápido crecimiento con la finalidad de cubrir en el menor tiempo posible dicha área intervenida, es por esto que muchas especies vegetales poseen baja densidad básica de la madera con fines de alcanzar mayor tamaño, luego inician a germinar las plantas con mediana densidad básica pero de crecimiento un poco más lento, hasta que finalmente se logra concretar a las especies de alta densidad y cuyo crecimiento es lento pero con dimensiones muy grandes, este comportamiento hace que un medio intervenido presenta diferentes valores de estructura vertical.

Es de suma importancia conocer la manera de distribución vertical de las especies vegetales, ya que en caso de las especies con mayor dominancia muestran predominancia impidiendo que los otros individuos tengan limitantes en emergen, dicho efecto se denomina onda de densidad (Aguilar, 2019) o en todo caso se observa la timidez de las plantas entre especies, el cual señala que son los espacios entre las copas de los indivicuos de la misma especie vegetal (Aguilar, 2020). Dicha información toma importancia en generar una herramienta y asignarles un manejo adecuado para el manejo de los bosques secundarios 
como es el caso del BRUNAS. El estudio tiene por objetivo determinar la estructura vertical e índice de valor forestal ecológico de la vegetación arbórea del Bosque Reservado en Tingo María.

\section{METODOLOGÍA}

\section{Características generales del área de estudio}

El estudio se llevó a cabo en dos parcelas permanentes de medición establecidas en el BRUNAS. Dicho bosque se encuentra en el distrito Rupa Rupa; provincia Leoncio Prado; región Huánuco. El bosque cuenta con una extensión de 217,22 ha, de las cuales solo 185 ha presentan cobertura boscosa (Puerta y Cárdenas, 2012). El clima del área de estudio, se consideró datos del año 2019, registrando precipitación anual de 3019,8 mm, humedad relativa de $82,5 \%$ y temperatura promedio anual de $25,7^{\circ} \mathrm{C}$

\section{Materiales y equipos}

Se utilizó los materiales para las mediciones dasométricas como son la wincha, cinta diamétrica; para reajustes de perímetro y subparcelas se utilizó jalones, rafia, tubos pvc; para la colecta y herborización de muestras se utilizó tijera telescópica, plumones, cinta masketing, bolsas, prensa botánica y periódico. Los equipos fueron: Cámara fotográfica, clinómetro, receptor GPS y brújula.

\section{Metodología}

\section{Descripción de la estructura vertical de la vegetación arbórea del BRUNAS}

Para la estructura vertical se recopiló información acerca de los métodos de evaluación y estratificación de los árboles. Asimismo, se planificó la medición de la altura total de los árboles con la finalidad de estratificar la vegetación arbórea de las PPMs, considerando tres niveles (baja, media y alta).

Descripción del Índice de Valor Forestal de la vegetación arbórea del BRUNAS

Para la estructura bidimensional se elaboraron formatos de evaluación para los datos requeridos como altura, diámetro de copa, diámetro de fuste. Estos datos fueron tomados de la evaluación realizada para la estrutura horizontal y vertical.

Construcción de las parcelas permanentes de muetreo (PPM)

Las parcelas permanentes de medición se establecieron el año 2002 con el fin de conocer la dinámica arbórea del BRUNAS, cuyas dimensiones son de $100 \mathrm{~m}$ x $100 \mathrm{~m}$, subdivididas en 25 subparcelas (Malleux, 1973, Gentry y Dodson, 1987, Mostacedo y Fredericksen, 2000). A las PPM y subparcelas de evaluación se les hizo un reajuste del perímetro, para ello se utilizó rafia y tubos PVC de 2 pulgadas de diámetro, las mismas que fueron colocados cada 20 metros. Actividad correspondiente a la numeración correlativa de los individuos que se encontraban al hacer el recorrido por cada subparcela teniendo en cuenta aquellos árboles con diámetros a la altura del pecho (dap) $\geq 10 \mathrm{~cm}$, los mismos que fueron recodificados utilizando clavos y placas de aluminio. 
Las placas fueron colocadas a $30 \mathrm{~cm}$ por encima del dap o pom. Las variables que se registraron fueron: altura total, diámetro a la altura del pecho (dap) y el diámetro de copa. Los árboles fueron identificados con la ayuda de especialistas en dendrología (Soto, 2016) teniendo en cuenta el género y especie. Las especies que no se pudieron identificar en ese momento, se colectaron las muestras (hojas, flores y frutos) para luego ser llevadas al herbario HOXA Selva Central.

\section{Análisis de datos}

Se utilizó las fórmulas citadas por Acosta et al. (2006), para determinar el valor de cobertura (VC), la posición sociológica absoluta (Psa) y posición sociológica relativa (Psr); para determinar el índice de espacio vital (IEV), se consideró las fórmulas utilizadas Burger (1939) y Serrada (2008), para el índice de valor forestal (IVF) se consideró lo indicado por Corella et al. (2001).
Para el análisis de los datos, se utilizó la estadística descriptiva (media o promedio, error estándar de la media y el coeficiente de variación).

\section{RESULTADOS}

Estructura vertical de la vegetación arbórea del brunas

\section{Posición sociológica de la vegetación arbórea del BRUNAS}

En la PPM I, se encontró 88 individuos con dap mayor o igual a $10 \mathrm{~cm}$ que se ubicaron en el estrato inferior $(<11,45 \mathrm{~m})$, mientras que en el estrato medio se registró 457 individuos y en el estrato superior (>22,9 m) se encontró 101 individuos; y la especie con mayor posición sociológica fue Senefeldera inclinata (Tabla 01).

\section{Tabla 1}

Posición sociológica de la vegetación arbórea en la PPM I del BRUNAS

\begin{tabular}{|c|c|c|c|c|c|c|}
\hline Especies & $\begin{array}{c}\text { E. I. } \\
\text { No/ha }\end{array}$ & $\begin{array}{l}\text { E. M. } \\
\mathrm{N}^{\mathrm{o}} / \mathrm{ha}\end{array}$ & $\begin{array}{l}\text { E. S. } \\
\mathrm{N}^{\circ} / \mathrm{ha}\end{array}$ & PSA & PSR & No/ha \\
\hline Senefeldera inclinata & 9 & 48 & 0 & 35,18 & 10,02 & 57 \\
\hline Parkia panurensis & 3 & 43 & 19 & 33,80 & 9,63 & 65 \\
\hline Casearia ulmifolia & 3 & 42 & 3 & 30,59 & 8,71 & 48 \\
\hline Pourouma minor & 5 & 21 & 5 & 16,32 & 4,65 & 31 \\
\hline Qualea amoena & 3 & 21 & 4 & 15,89 & 4,53 & 28 \\
\hline Virola pavonis & 2 & 18 & 7 & 14,10 & 4,02 & 27 \\
\hline Schizocalyx peruvianus & 15 & 15 & 2 & 12,97 & 3,69 & 32 \\
\hline Helicostylis tomentosa & 6 & 15 & & 11,43 & 3,26 & 21 \\
\hline Laetia procera & 1 & 13 & 4 & 9,96 & 2,84 & 18 \\
\hline Theobroma subincanum & 1 & 12 & & 8,63 & 2,46 & 13 \\
\hline Otras 97 especies & 40 & 209 & 57 & & & 306 \\
\hline Total & 88 & 457 & 101 & & & 646 \\
\hline
\end{tabular}

Nota: E. I.Estrato inferior, E. M.: medio, E. S.: superior. PSA: Posición sociológica absoluta, PSR: PS relativa 
En caso de la PPM IV, no se registró relación alguna entre la cantidad de individuos e intervalos de los estratos, encontrándose 98 individuos en el estrato inferior ( $<13 \mathrm{~m}), 371$ individuos en el estrato medio y 75 individuos en el estrato superior ( $>26 \mathrm{~m})$. Entre las 10 especies con mayor posición sociológica se encontraban: Senefeldera inclinata con mayor representatividad $(31,90 \%)$, seguido de Cecropia sciadophylla, Pourouma minor, Otoba parvifolia, Schizocalyx peruvianus, Dacryodes nitens, Tapirira guianensis, Psychotria levis, Pseudolmedia laevis y Theobroma subincanum (Tabla 02).

\section{Tabla 2}

Posición sociológica de la vegetación arbórea en la PPM IV del BRUNAS

\begin{tabular}{|c|c|c|c|c|c|c|}
\hline Especies & $\begin{array}{c}\text { E. I. } \\
\mathrm{N}^{\circ} / \mathrm{ha}\end{array}$ & $\begin{array}{l}\text { E. M. } \\
\text { No/ha }\end{array}$ & $\begin{array}{c}\text { E. S. } \\
N^{\circ} / h a\end{array}$ & PSA & PSR & $\mathrm{N} / \mathrm{ha}$ \\
\hline Senefeldera inclinata & 32 & 123 & 0 & 89,65 & 31,90 & 155 \\
\hline Cecropia sciadophylla & 1 & 13 & 3 & 9,46 & 3,37 & 17 \\
\hline Pourouma minor & 3 & 9 & 11 & 8,19 & 2,92 & 23 \\
\hline Otoba parvifolia & 2 & 10 & 3 & 7,59 & 2,70 & 15 \\
\hline Schizocalyx peruvianus & 7 & 8 & 0 & 6,72 & 2,39 & 15 \\
\hline Dacryodes nitens & 2 & 8 & 2 & 6,09 & 2,17 & 12 \\
\hline Tapirira guianensis & 3 & 8 & 0 & 6,00 & 2,13 & 11 \\
\hline Psychotria levis & 4 & 7 & 0 & 5,49 & 1,96 & 11 \\
\hline Pseudolmedia laevis & & 8 & 0 & 5,46 & 1,94 & 8 \\
\hline Theobroma subincanum & 4 & 6 & 1 & 4,95 & 1,76 & 11 \\
\hline Otras 107 especies & 40 & 171 & 55 & & & 266 \\
\hline Total & 98 & 371 & 75 & & & 544 \\
\hline
\end{tabular}

Nota: E. I Estrato inferior, E. M.: estrato medio, E. S.: estrato superior. PSA: Posición sociológica absoluta, PSR: posición sociológica relativa

\section{Índice de espacio vital de la vegetación arbórea del BRUNAS}

Teniendo en consideración las 10 especies de la PPM I con mayor relación entre el diámetro de la copa con el diámetro a la altura del pecho, la especie Miconia punctata sobresalió con un valor de 0,98 , seguida de Eugenia egensis, Rauvolfia sprucei, Pseudolmedia laevis, Miconia dolichorrhyncha, Lacistema nena, Brosimum guianense, Miconia chrysophylla, Tetragastris panamensis y Micropholis guyanensis con valores inferiores. Además, es notorio que algunas especies solo estuvieron representadas por un individuo, motivo por el cual se registró un coeficiente de variación nulo (Tabla 03). 


\section{Tabla 3}

Índice de espacio vital de la vegetación arbórea en la PPM I del BRUNAS

\begin{tabular}{|c|c|c|c|}
\hline Especies & Media & Error estándar & $\mathrm{CV}(\%)$ \\
\hline Miconia punctata & 0,98 & 0,00 & 0,00 \\
\hline Eugenia egensis & 0,52 & 0,00 & 0,00 \\
\hline Rauvolfia sprucei & 0,45 & 0,01 & 4,33 \\
\hline Pseudolmedia laevis & 0,45 & 0,05 & 22,46 \\
\hline Miconia dolichorrhyncha & 0,44 & 0,07 & 31,28 \\
\hline Lacistema nena & 0,43 & 0,09 & 44,90 \\
\hline Brosimum guianense & 0,42 & 0,00 & 0,00 \\
\hline Miconia chrysophylla & 0,40 & 0,10 & 34,34 \\
\hline Tetragastris panamensis & 0,40 & 0,03 & 8,98 \\
\hline Micropholis guyanensis Subsp. Guyanensis & 0,40 & 0,10 & 45,43 \\
\hline Especies & Media & Error estándar & $\mathrm{CV}(\%)$ \\
\hline Miconia punctata & 0,98 & 0,00 & 0,00 \\
\hline Eugenia egensis & 0,52 & 0,00 & 0,00 \\
\hline Rauvolfia sprucei & 0,45 & 0,01 & 4,33 \\
\hline Pseudolmedia laevis & 0,45 & 0,05 & 22,46 \\
\hline Miconia dolichorrhyncha & 0,44 & 0,07 & 31,28 \\
\hline Lacistema nena & 0,43 & 0,09 & 44,90 \\
\hline Brosimum guianense & 0,42 & 0,00 & 0,00 \\
\hline Miconia chrysophylla & 0,40 & 0,10 & 34,34 \\
\hline Tetragastris panamensis & 0,40 & 0,03 & 8,98 \\
\hline Micropholis guyanensis Subsp. Guyanensis & 0,40 & 0,10 & 45,43 \\
\hline
\end{tabular}

Nota: CV coeficiente de variación. Datos referidos a cero (0) debido a la presencia de un solo individuo

Para el caso de la PPM IV, se registraron que las 10 primeras especies vegetales con mayor índice de espacio vital fueron: Hirtella racemosa var. Hexandra con un valor de 0,79 , seguido de las especies con menores valores que fueron: Miconia punctata,
Brosimum utile, Ficus casapiensis, Eugenia indet indet, Protium amazonicum, Miconia indet, Aniba guianensis, Bellucia pentamera y Chimarrhis hookeri (Tabla 04).

\section{Tabla 4}

Índice de espacio vital de la vegetación arbórea en la PPM IV del BRUNAS

\begin{tabular}{|c|c|c|c|}
\hline Especies & Media & Error estándar & $\mathrm{CV}(\%)$ \\
\hline Hirtella racemosa var. hexandra & 0,79 & 0,00 & 0,00 \\
\hline Miconia punctata & 0,65 & 0,00 & 0,00 \\
\hline Brosimum utile & 0,63 & 0,00 & 0,00 \\
\hline Ficus casapiensis & 0,63 & 0,00 & 0,00 \\
\hline Eugenia indet indet & 0,54 & 0,00 & 0,00 \\
\hline Protium amazonicum & 0,52 & 0,00 & 0,00 \\
\hline
\end{tabular}




\begin{tabular}{llll}
\hline Miconia indet & 0,52 & 0,00 & 0,00 \\
Aniba guianensis & 0,49 & 0,00 & 0,00 \\
Bellucia pentamera & 0,49 & 0,00 & 0,00 \\
Chimarrhis hookeri & 0,49 & 0,00 & 0,00 \\
\hline
\end{tabular}

Nota: CV coeficiente de variación. Datos referidos a cero (0) debido a la presencia de un solo individuo

Índice de Valor Forestal (estructura bidimensional) de la vegetación arbórea del bosque reservado de la Universidad Nacional Agraria de la Selva

En la PPM I, se determinó que la especie forestal Parkia panurensis registró 39,73\% respecto al índice de valor forestal, siendo superior el valor encontrado para dicho PPM, las nueve especies forestales restantes con mayores valores fueron: Senefeldera inclinata (21,34\%), Casearia ulmifolia $(20,22 \%)$, Virola pavonis $(13,45 \%)$, Pourouma minor $(13,25 \%)$,

\section{Tabla 5}

Índice de valor forestal de la vegetación arbórea en la PPM I del BRUNAS

\begin{tabular}{lccccccc}
\hline \multirow{2}{*}{ Especies } & \multicolumn{2}{c}{ Diámetro } & \multicolumn{2}{c}{ Altura } & \multicolumn{2}{c}{ Cobertura } & \multirow{2}{*}{ IVF } \\
\cline { 2 - 6 } & Abs. & Rel. & Abs. & Rel. & Abs. & Rel. & \\
\hline Parkia panurensis & 0,82 & 13,04 & 0,65 & 11,64 & 5,44 & 15,04 & 39,73 \\
Senefeldera inclinata & 0,42 & 6,60 & 0,43 & 7,74 & 2,53 & 6,99 & 21,34 \\
Casearia ulmifolia & 0,42 & 6,67 & 0,39 & 7,02 & 2,36 & 6,53 & 20,22 \\
Virola pavonis & 0,28 & 4,38 & 0,26 & 4,62 & 1,61 & 4,45 & 13,45 \\
Pourouma minor & 0,28 & 4,41 & 0,27 & 4,79 & 1,47 & 4,06 & 13,25 \\
Qualea amoena & 0,21 & 3,32 & 0,26 & 4,68 & 0,86 & 2,38 & 10,38 \\
Helicostylis tomentosa & 0,18 & 2,84 & 0,16 & 2,88 & 0,95 & 2,62 & 8,35 \\
Jacaranda copaia & 0,17 & 2,74 & 0,11 & 2,00 & 1,17 & 3,23 & 7,96 \\
Laetia procera & 0,17 & 2,62 & 0,16 & 2,88 & 0,79 & 2,19 & 7,69 \\
Schizocalyx sterculioides & 0,15 & 2,38 & 0,15 & 2,76 & 0,44 & 1,21 & 6,35 \\
\hline Otras 97 especies & 3,21 & 51,00 & 2,74 & 48,98 & 18,54 & 51,30 & 151,28 \\
\hline
\end{tabular}

Nota: IVF índice de valor forestal

El índice de valor forestal (acumulados del diámetro altura y cobertura relativas) determinado para las especies de la PPM IV del BRUNAS, indica que la especie forestal Senefeldera inclinata registró un valor de $68,28 \%$, seguido de las nueve especies con mayor valor forestal como son: Pourouma minor,
Qualea amoena (10,38\%), Helicostylis tomentosa $(8,35 \%)$, Jacaranda copaia $(7,96 \%)$, Laetia procera $(7,69 \%)$ y Schizocalyx sterculioides $(6,35 \%)$, que en conjunto alcanzaron $108,99 \%$ de dicho índice. En caso de las 97 especies pertenecientes a la vegetación arbórea restantes en la PPM I, se determinó que el IVF acumulado ascendió de $151,28 \%$, con $51,00 \%$ de diámetro relativo, $48,98 \%$ de altura relativa y $51,30 \%$ de cobertura relativa (Tabla 05 ). 


\section{Tabla 6}

Índice de valor forestal de la vegetación arbórea en la PPM IV del BRUNAS

\begin{tabular}{lccccccc}
\hline \multirow{2}{*}{ Especies } & \multicolumn{2}{c}{ Diámetro } & \multicolumn{2}{c}{ Altura } & \multicolumn{2}{c}{ Cobertura } & \multirow{2}{*}{ IVF } \\
\cline { 2 - 6 } & Abs. & Rel. & Abs. & Rel. & Abs. & Rel. \\
\hline Senefeldera inclinata & 1,30 & 20,83 & 1,31 & 25,33 & 8,06 & 22,12 & 68,28 \\
Pourouma minor & 0,34 & 5,48 & 0,28 & 5,42 & 2,20 & 6,03 & 16,93 \\
Cecropia sciadophylla & 0,22 & 3,55 & 0,19 & 3,72 & 1,89 & 5,20 & 12,48 \\
Dacryodes nitens & 0,19 & 3,04 & 0,12 & 2,36 & 1,30 & 3,56 & 8,96 \\
Hevea guianensis & 0,21 & 3,43 & 0,14 & 2,66 & 1,00 & 2,76 & 8,85 \\
Otoba parvifolia & 0,18 & 2,89 & 0,15 & 2,85 & 1,02 & 2,80 & 8,53 \\
Cedrelinga cateniformis & 0,18 & 2,87 & 0,07 & 1,38 & 0,99 & 2,73 & 6,97 \\
Tapirira guianensis & 0,13 & 2,07 & 0,09 & 1,72 & 0,95 & 2,60 & 6,40 \\
Osteophloeum platyspermum & 0,16 & 2,50 & 0,09 & 1,69 & 0,79 & 2,16 & 6,35 \\
Guatteria guentheri & 0,15 & 2,46 & 0,11 & 2,07 & 0,59 & 1,63 & 6,15 \\
\hline Otras 107 especies & 3,17 & 50,88 & 2,62 & 50,80 & 17,63 & 48,41 & 150,09 \\
\hline
\end{tabular}

\section{DISCUSIÓN}

La posición sociológica en la PPM I indica que en el estrato inferior se encontraban $13,62 \%$ de los árboles, $70,59 \%$ para el estrato medio y $15,79 \%$ en el estrato superior, mientras que la PPM IV presentaba $18,01 \%$ en el estrato inferior, $66,91 \%$ en el estrato medio y $15,07 \%$ en el estrato superior, esto se debe a la complejidad la estructura del bosque y que el BRUNAS presenta una etapa de sucesión por presentar perturbaciones anteriormente; al respecto Richards (1952) indica que, sobre estratos se encuentra el dosel de árboles grandes que tiene entre 25 y 40 metros de altura dependiendo del lugar. Sobre el dosel hay aisladamente gigantescos árboles emergentes que pueden llegar a medir hasta 70 metros en las partes más exuberantes de la selva, creciendo sobre toda la diversidad de tamaños de árboles hay una asombrosa abundancia de plantas criptógamas, trepadoras, epífitas y árboles estranguladores que contribuyen a la complejidad estructural de la comunidad; dichos valores de tamaños difieren del BRUNAS por la edad de la misma.
Esta dinámica sobre la posición sociológica se encuentra relacionada con la luminosidad de las dos parcelas permanentes, debido que para Krebs (1986), la estructura vertical está asociada con una disminución de la luminosidad. Donde la competencia por la luz es un factor crítico en la determinación de la estratificación del bosque. La luz es un recurso muy importante y la competencia entre plantas para llegar a la luz es fuerte, la planta ganadora no es necesariamente la planta con mayor follaje, sino aquella cuyo follaje presenta la mejor disposición para interceptar la luz, la altura de la planta juega un papel muy importante para la captación de luz. Los resultados de la posición sociológica dependerán de múltiples factores del medio, ya que resultados diferentes a lo obtenido del BRUNAS lo reportan Zarco-Espinosa et al. (2010) en la vegetación arbórea en el parque estatal Agua Blanca (México), identificando una clara discontinuidad en la distribución de alturas: $87 \%$ de individuos en el estrato inferior $(<9 \mathrm{~m})$ y $13 \%$ en el superior (>9 m), la cual coincidía con lo obtenido por Godínez-Ibarra \& López-Mata (2002) en una selva mediana subperennifolia de Veracruz (México), 
donde la distribución de individuos por su altura mostró valores cercanos a $88 \%$ en el estrato inferior y a $12 \%$ en los superiores.

Para el índice de espacio vital en la vegetación arbórea del BRUNAS, se reporta que las especies con menor cantidad de individuos alcanzaron los mayores valores de dicho índice, comportamiento principal que se le atribuye a la competencia entre la vegetación arbórea, ya que Serrada (2008) añade que, este índice se basa en considerar dos indicadores básicos del desarrollo o morfología de un árbol, consecuencia de la espesura en que vive o ha vivido. Autores como Arias (2005), añaden que este índice crece a medida que el árbol engrosa en diámetro, y las especies encontradas como Miconia punctata $(10,49 \mathrm{~cm}$ de dap y 10,30 m del diámetro de copa) en la PPM I y Hirtella racemosa var. hexandra $(11,36 \mathrm{~cm}$ de dap y 9,00 $\mathrm{m}$ de diámetro de copa) en la PPM IV presentaban copas muy pequeñas en relación al dap y además estuvieron representadas por un solo individuo.

El índice de valor forestal en el BRUNAS, describe que la especie Parkia panurensis registró 39,73\% respecto al índice de valor forestal en la PPM I, mientras que Senefeldera inclinata registró un valor de 68,28\% en la PPM IV, dicha variación se le puede atribuir a las particularidades de la ubicación de las parcelas.

Se registró 117 especies en la PPM IV, mientras que en la PPM I hubo 107 especies, las cueles fueron superiores a lo reportado por Blas (2002) al evaluar una parcela permanente en el BRUNAS, donde registró 97 especies con 67 géneros distribuidos en 32 familias. Osinfor (2014) en bosques de selva alta (Bosque Reservado de la Universidad Nacional
Agraria de la Selva) identificó las siguientes especies arbóreas representativas como: Rollinia peruviana Diels., Calycophyllum megistocaulum (K. Krause) C.M. Taylor., Cecropia sciadophylla Mart., Osteophloem platyspermun (Spruce ex A. DC. Warb., Virola pavonis (A.DC.) A.C. Sm., Jacaranda copaia (Aubl.) D. Don., Senefeldera macrophylla Ducke (especie diferente a lo reportado en el estudio), Cavanillesia umbellata Ruiz \& Pav., Bellucia pentamera Naudin., Caryodendron orinocense $\mathrm{H}$. Karst., Batocarpus orinocensis H. Karst., Brosimun rubescens Tub., Macrolobium gracile Spruce ex Benth., Guarea kunthiana A. Juss., Pourouma bicolor Mart., Pourouma minor Benoist., Inga pezizifera Benth., Hevea guianensis Aubl., Sapium glandulosum (L.) Morong., Ochroma pyramidale (Cav. ex Lam.) Urb. y Tapirira guianensis Aubl.

En la parcela I se reporta a siete especies de las 10 de mejor posición sociológica que se encontraban en los tres estratos, mientras que en caso de la parcela IV se reporta a cinco especies, dicha distribución de individuos en los estratos lo reportan García et al. (2019) en especies coníferas, denominándolos como especies con distribución vertical continua, con el cual estas especies vienen asegurando la permanencia en el tiempo dentro de su composición y la estructura del BRUNAS. Un caso muy particular ocurre con la especie Senefeldera inclinata, el cual no aparece en el estrato superior para ambas parcelas evaluadas, esto se le puede atribuir al porte de dicho árbol, siendo ausente en el estrato superior de muchos tipos de bosques.

Una de las características muy particular del BRUNAS es que a pesar de que presentaba individuos de Cedrelinga cateniformis con diámetro 
y altura total muy relevantes, no se logra ubicar entre los de mayor posición sociológica debido a que solamente fueron pocos árboles y aún no se logra garantizar la sobrevivencia de la regeneración natural debido a la alta dinámica en esta área de sucesión, así como la extracción para su comercialización por las personas ajenas a la universidad, caso contrario se reportó para Parkia panurensis debido a su rápido crecimiento que lo caracteriza (Luccini et al., 2018) y al crecimiento en suelos intervenidos observados en la selva amazónica de Loreto. Una vez que el árbol haya dominado y se encuentra en el estrato superior, generan mediante su copa un grupo de árboles suprimidos que son de la misma especie provenientes de la regeneración natural u otras especies que debido a su sombreamiento y al poco espacio para emerger les limitan su crecimiento, siendo dicho efecto consecuente del estrato medio hacia los individuos que se encuentran en el estrato inferior, este acontecimiento es denominado como onda de densidad que se desvanece mientras más se aleja del centro de la copa de los árboles hacia la parte lateral de la copa, por otra parte, en caso de ser la misma especie, se observa el fenómeno conocido como timidez de las pantas manteniedo separadas las copas de los árboles el cual se ocasiona por los vientos que hay en la zona (Aguilar, 2020).

Al comparar los valores de los estratos, se muestra cierta similitud al comparar con un bosque templado (Dávila et al., 2019), esto posiblemente es atribuido a la edad del bosque, el cual, en caso del BRUNAS, se encuentra en transición hacia un bosque primario y los individuos estarían alcanzando mayor altura total. Además, en la parcela IV, se observó mayores individuos en el estrato medio, seguido del estrato inferior; cuando un bosque es sometido al manejo como actividades de la corta de lianas, se observa que hay mayor número de plantas en el estrato inferior debido a la proliferación de la regeneración natural (García et al., 2019), a pesar que hasta cierto edad nomás se oberva abundante regeneración debido a que por la competencia entre individuos, algunas plantas mueren a pesar de tener un tamaño considerable.

\section{CONCLUSIONES}

La estructura vertical de la vegetación arbórea del BRUNAS, para la PPM I, presentó 88 individuos en el estrato inferior ( $<11,45 \mathrm{~m}$ de altura), en el estrato medio registró 457 individuos y en el estrato superior (> 22,9 $\mathrm{m}$ de altura) hubo 101 individuos, siendo Senefeldera inclinata $(10,02 \%)$ con mayor posición sociológica y Miconia punctata sobresalió con mayor índice de espacio vital $(0,98)$; en caso de la PPM IV, se registró 98 individuos en el estrato inferior ( $<13 \mathrm{~m}$ de altura total), 371 individuos en el estrato medio y 75 individuos en el estrato superior (> $26 \mathrm{~m}$ de altura total), siendo Senefeldera inclinata con mayor posición sociológica $(31,90 \%)$ e Hirtella racemosa var. Hexandra con mayor índice de espacio vital $(0,79)$.

En la estructura bidimensional de la vegetación arbórea del BRUNAS, la especie Parkia panurensis registró 39,73\% del índice de valor forestal en la PPM I y Senefeldera inclinata registró 68,28\% para la PPM IV. 


\section{REFERENCIAS}

[1] Acosta, V., Araujo, P., Iturre, M. (2006). Caracteres estructurales de las masas. Serie didáctica sociología vegetal y fitogeografía forestal, $\mathrm{N}^{\circ}$ 22. Santiago del Estero, Argentina, UNSE.

[2] Aguilar, J.M.E. (2019). Diseños 'Nelder' en la investigación forestal: una revisión. Avances en Investigación Agropecuaria, 23(1), 7-25. http://ww.ucol.mx/revaia/portal/pdf/2019/en ero/1.pdf

[3] Aguilar, J.M.E., Cabrera, N., Barrios, B., y Loeza, J. M. (2020). Efecto de onda y fenómeno de timidez en bosques homogéneos de Alnus acuminata. Madera y Bosques, 26(1), 1-14. https://myb.ojs.inecol.mx/index.php/myb/art icle/view/e2611939/2058

[4] Arias, D. (2005). Morfometría del árbol en plantaciones forestales tropicales. Revista Forestal Kurú, 2(5):1-13.

[5] Baró, F., Chaparro, L., Gómez-Baggethun, E., Langemeyer, J., Nowak, D. J., \& Terradas, J. (2014). Contribution of ecosystem services to air quality and climate change mitigation policies: The case of urban forests in Barcelona, Spain. Ambio, 43(4), 466-479. https://doi.org/10.1007/s13280014-0507-x

[6] Beckett, K.P., Freer-Smith, P.H. \& Taylor, G. (2000). Effective tree species for local air quality management. Journal of Arboriculture, 26(1), 12-19.
[7] Blas, D. (2002). Establecimiento y evaluación de parcelas permanentes de medición en el Bosque Reservado de la Universidad Nacional Agraria de la Selva [Tesis de titulo, Universidad Nacional Agraria de la Selva, Perú].

[8] Burger, H. (1939). Baumkrone und zuwachs in zwei hiebsreifen fichtenbeständen. Mitteilungen der Schweizerischen Anstalt für das Forstliche Versuchenwesen, 21:147-176.

[9] Burkhart, H.E. \& Tomé, M. (2012). Modeling Forest Trees and Stands. Dordrecht: Springer Netherlands. https://doi.org/10.1007/978-90-481-3170-9

[10] Corella, J.F., Valdez, H.J.I., Cetina, A.V.M., González, C.F.V., Trinidad, S.A., Aguirre, R.J.R. (2001). Estructura forestal de un bosque de mangles en el noreste del estado de Tabasco, México. Ciencia Forestal en México, 26(90):73-102.

[11] Dávila, M.A., Aguirre, O.A., Jurado, E., Treviño, E., González, M., y Trincado, G. (2019). Estructura y diversidad de especies arbóreas en bosques templados de San Luis Potosí, México. Ecosist. Recur. Agropec., $6(18)$, 399-409. http://www.scielo.org.mx/pdf/era/v6n18/200 7-901X-era-6-18-399.pdf

[12] García, S.A., Narváez, R., Olivas, J.M., y Hernández, J. (2019). Diversidad y estructura vertical del bosque de pino-encino en Guadalupe y Calvo, Chihuahua. Revista Mexicana de Ciencias Forestales, 10(53), 4163. 
http://www.scielo.org.mx/pdf/remcf/v10n53

12007-1132-remcf-10-53-41.pdf

[13] Gentry, H. y Dodson, C. (1987). Contribution of non-trees to species richness of tropical rain forest. Biotrópica, 19:149-156.

[14] Gestión. (2020). La deforestación en la amozonia

peruana.

https://gestion.pe/peru/deforestacion-en-laamazonia-peruana-se-redujo-287-durante-lacuarentena-noticia/?ref=gesr

[15] Godínez-Ibarra, O. y López-Mata, L. (2002). Estructura, composición, riqueza y diversidad de árboles en tres muestras de selva mediana subperennifolia. Anales del Instituto de Biología. Universidad Nacional Autónoma de México. Serie Botánica, 73(2):283-314.

[16] Guerra, F., García, A., Martínez, M.A. (2020). Evaluación de la resiliencia ecológica de los bosques tropicales secos: una aproximación multiescalar. Madera y Bosques, 26(3), 1-19. http://www.scielo.org.mx/pdf/mb/v26n3/244 8-7597-mb-26-03-e2631983.pdf

[17] Janhäll, S. (2015). Review on urban vegetation and particle air pollution $\mathrm{e}$ Deposition and dispersion. Atmospheric Environment, 105, 130-137. https://doi.org/10.1016/j.atmosenv.2015.01. 052

[18] Korhonen, L., Vauhkonen, J., Virolainen, A., Hovi, A., \& Korpela, I. (2013). International Journal of Remote Sensing Estimation of tree crown volume from airborne lidar data using computational geometry Estimation of tree crown volume from airborne lidar data using computational geometry. International Journal of Remote Sensing, 34(20), 72367248 .

https://doi.org/10.1080/01431161.2013.8177 15

[19] Krebs, C. (1986). Ecología. Madrid España. JOSMAR S.A.

[20] Li, Y., Wang, X.R. \& Huang, C.L. (2011). Key street tree species selection in urban areas. African Journal of Agricultural Research, 6(15), 3539-3550. https://doi.org/10.5897/AJAR11.461

[21] Luccini, E., Flores, M., Ramírez, K., PérezPinedo, H., Herrera, L., Parodi, M., Matar, M., Gómez-Insausti, J., Barrea, L., Mechini, M., Calcagno, G. (2018). Análisis comparativo de la captura y almacenamiento de $\mathrm{CO} 2$ por especies vegetales de tres ecosistemas en Perú. Energeia, 15(15), 1321.

https://repositorio.uca.edu.ar/bitstream/1234 56789/5870/1/analisis-comparativo-capturaalmacenamiento.pdf

[22] Malleux, J. (1973). Análisis de dispersión de 10 especies forestales de los Bosques de Nueva Italia. Revista Forestal del Perú. Universidad Nacional Agraria La Molina. $5(1-2)$.

[23] Mori, J., Hanslin, H. M., Burchi, G., \& Sæbø, A. (2015). Particulate matter and element accumulation on coniferous trees at different distances from a highway. Urban Forestry and Urban Greening, 14(1),170-177. https://doi.org/10.1016/j.ufug.2014.09.005 
[24] Mostacedo, B. y Fredericksen, T. (2000). Manual de métodos básicos de muestreo y análisis en Ecología Vegetal. Manual BOLFOR. Santa Cruz, Bolivia. http://www.bio-

nica.info/biblioteca/mostacedo2000ecologia vegetal.pdf.

[25] Nowak, D.J., \& Heisler, G.M. (2010). Air Quality Effects of Urban Trees and Parks.

[26] O'Hara K.L., Latham, P.A. y Valappil N. (1995). Parameters for describing stand structure. In: Skovsgaard, J.P., Burkhart, H.E. (Eds.), Recent Advances in Forest Mensuration and Growth and Yield Research. Ministry of Environment and Energy, Danish Forest and Landscape Research Institute, Hørsholm, Denmark, pp. 134-145.

[27] Osinfor. 2014. Organismo de supervisión de los Recursos forestales y de Fauna Silvestre, Perú. Fichas de identificación de especies forestales maderables de la zona de Tingo María. Curso taller del 24 de febrero al 03 de marzo 2014 "Bases dendrológicas y silviculturales para el manejo responsable de los bosques tropicales Tingo María Huánuco - Perú”. (Serie técnica No 5), 1:151.

[28] Ramos, B. (2004). Estructura y composición de un paisaje boscoso fragmentado: Herramienta para el diseño de estrategias de conservación de la biodiversidad. Composición florística y su estructura en un bosque húmedo montano al noroeste del Parque Nacional Madidi [Tesis de grado de maestro, Turrialba, Costa Rica. PEDCCATIE].

[29] Räsänen, J. V., Holopainen, T., Joutsensaari, J., Ndam, C., Pasanen, P., Rinnan, Å., \& Kivimäenpää, M. (2013). Effects of speciesspecific leaf characteristics and reduced water availability on fine particle capture efficiency of trees. Environmental Pollution, 183 , 64-70. https://doi.org/10.1016/j.envpol.2013.05.015

[30] Richards, W. 1952. The tropical rain forest. Cambridge: Cambridge University Press.

[31] Saavedra-Romero, L.L., Alvarado-Rosales, D., Hernández-De La Rosa, P., MartínezTrinidad, T., Mora-Aguilera, G. \& VillaCastillo, J. (2016). Crown condition, a health indicator in urban trees of the San Juan de Aragon Park, Mexico City. Madera y Bosques, 22(2), 15-27.

[32] Savard, J.P.L., Clergeau, P. \& Mennechez, G. (2000). Biodiversity concepts and urban ecosystems. Landscape and Urban Planning, 48(3-4), 131-142. https://doi.org/10.1016/S0169-

2046(00)00037-2

[33] Schomaker, M.E., Zarnoch, S.J., Bechtold, W.A., Latele, D.J., Burkman, W.G. \& Cox, S.M. (2007). Crown-Condition Classification: A Guide to Data Collection and Analysis. USDA Gen. Tech. Rep. SRS102. U.S. Department of Agriculture Forest Service, Asheville, NC., 92.

[34] Serrada, R. (2008). Selvicultura de Bosques. Servicio de Publicaciones. EUIT Forestal. Madrid, España. 
[35] Servindi. (2016). Servicios de Comunicación Intercultural.

https://www.servindi.org/actualidadnoticias/06/07/2016/en-tres-anos-sedeforestaron-25-mil-hectareas-en-selva-dehuanuco

[36] Soto, Y.C. (2016). Inventario dendrológico de una parcela permanente de medición del Bosque Reservado de la Universidad Nacional Agraria de la Selva - Tingo María, Perú [Tesis de titulo, Universidad Nacional Agraria de la Selva, Perú].

[37] Troxel, B., Piana, M., Ashton, M.S. \& Murphy-Dunning, C. (2013). Relationships between bole and crown size for young urban trees in the northeastern USA. Urban Forestry \& Urban Greening. https://doi.org/10.1016/j.ufug.2013.02.006

[38] Valerio, J. y Salas, C. (2001). Selección de prácticas silviculturales para bosques tropicales, manual técnico. $2^{\circ}$ Edición. Bolfor, Cobija. Bolivia.

[39] Zarco-Espinosa, V., Valdez-Hernández, J., Ángeles-Pérez, G., Castillo-Acosta, O. (2010). Estructura y diversidad de la vegetación arbórea del parque estatal Agua blanca, Macuspana, Tabasco. Revista Uciencia, 26(1):1-17. 